BERYLLIUM AND LITHIUM ABUNDANCES IN STARS WITH A RANGE OF METALLICITIES

RAMON J. GARCIA LOPEZ, EDUARDO L. MARTIN, RAFAEL REBOLO, AND JOHN E. BECKMAN

Instituto de Astrofísica de Canarias, E-38200, La Laguna, Spain

CONAL D. MCKEITH

Department of Pure and Applied Physics, The Queen's University of Belfast

JOHN K. WEBB

University of New South Wales

YAKOV V. PAVLENKO

The Principal Observatory of Ukraine

ABSTRACT We present new beryllium abundances for three unevolved stars with $[\mathrm{Fe} / \mathrm{H}]$ in the range -0.4 to -1.6 . The Be II doublet is detected in the three stars. A spectral synthesis analysis has been conducted to derive the abundances. We briefly discuss their $\mathrm{Be}$ and $\mathrm{Li}$ abundances, together with a wide sample of stars from the literature, in the context of mixing processes below the convection zone.

\title{
INTRODUCTION
}

Measurements of beryllium abundances in $F$ and $G$ stars with different metallicities provide information on mixing processes below their convective envelopes. Like lithium, beryllium is a fragile element which is destroyed in stellar interiors at relatively low temperatures (Be burns at $\sim 3.5 \times 10^{6} \mathrm{~K}$ while $\mathrm{Li}$ burns at $\left.2.5 \times 10^{6} \mathrm{~K}\right)$. These temperatures are typical of the layers close to the base of the convective zone in $F$ and $G$ stars. The measurement of lithium in such stars has shown the important effect of metallicity on the efficiency of sub surface particle transport processes. The stars with $[\mathrm{Fe} / \mathrm{H}]<-0.5$ are able to preserve a large amount of lithium in their convective zones. On the contrary, stars with solar metallicity strongly deplete this element during their evolution, showing both an abundance decrease with decreasing mass and the "Li gap" found in middle F-type stars. There are a large number of lithium measuarements available in the literature (relatively easy to achieve using the resonance doublet at $\lambda 6708 \AA$ ), but only very few of beryllium. This poor situation for beryllium determinations is mainly due to the fact that the only observable Be feature (the Be II resonance doublet at $\lambda 3131 \AA$ ) lies in a very dense spectral region 
close to the atmospheric transmission cut-off. This paper presents new beryllium abundances for three stars in the metallicity range -0.4 to -1.6 . In order to use these $\mathrm{Be}$ abundances to constrain the role of mixing processes below the convection zone, we have incorporated them in a larger sample of stars from the literature with known $\mathrm{Be}$ and $\mathrm{Li}$ abundances.

\section{OBSERVATIONS AND ANALYSIS}

The observations of two of the stars (HD132475 and HD38510) were carried out using the UCL echelle spectrograph attached to the AAT, providing a dispersion of $0.024 \AA /$ pixel at $\lambda 3130 \AA$. The third star (HD 165908) was observed with the LACUB (Instituto de Astrofísica de Canarias-University of Belfast) echelle spectrograph, at the $2.5 \mathrm{~m}$ Nordic Optical Telescope on La Palma. The dispersion obtained in this case was $0.017 \AA /$ pixel. These observations belong to a large programme designed to measure light element abundances to test nucleosynthesis and mixing processes. We carried out a standard data reduction using the IRAF package.

To derive the beryllium abundance for each star we performed a spectral synthesis in order to fit the profile of the weaker line of the Be II doublet, which is clearly resolved in our spectra. Details on the stellar parameters and analysis procedure are given in Rebolo et al. (1992). Our error bar estimate for a beryllium determination is $0.3 \mathrm{dex}$, which includes errors in the synthesis fit as well as uncertainties in the adopted stellar parameters.

\section{DISCUSSION AND CONCLUSIONS}

Our results can be summarized as follows:

We have detected the Be II doublet in the three stars observed. The abundances inferred are in the range $\log \mathrm{N}(\mathrm{Be})=0.6-0.8$ (in the scale $\log \mathrm{N}(\mathrm{H})=$ 12).

In Figure Ia we plot the beryllium abundance versus effective temperature for the compiled Be data, while in Figure Ib we show lithium abundances versus the same parameter. In both figures we have divided the stars into three groups as a function of their metallicity: (i) $[\mathrm{Fe} / \mathrm{H}]>-0.5$, (ii) $-0.5>[\mathrm{Fe} / \mathrm{H}]>-1.6$, and (iii) $[\mathrm{Fe} / \mathrm{H}]<-1.6$. In group (i) there is no evidence of Be depletion, except in the case of the hottest $\operatorname{star}(6900 \mathrm{~K})$. Their $\mathrm{Li}$ abundances show the well known decline towards lower temperatures. The absence of Be depletion sets constraints on the mechamisms which cause the $\mathrm{Li}$ depletion in the lower Main Sequence (pre-Main Sequence depletion, mixing due to rotation and angular momentum loss, stellar winds, etc.). On the other hand, the hottest star in the sample shows both $\mathrm{Be}$ and $\mathrm{Li}$ depletion. This object is located in the range of $\mathrm{F}$. type stars where the $\mathrm{Li}$ gap is present. Several mechanisms have been proposed to explain this phenomenon, and some of these predict also a Be depletion for the stars located in the $\mathrm{Li}$ gap (see García López and Spruit 1991 and references therein).

The stars belonging to group (ii) show fairly constant abundances for $\mathrm{Li}$ and $\mathrm{Be}$, and are in an effective temperature range of 5500-5900 $\mathrm{K}$. Their Be abundances are slightly below those of group (i), and considerably above those 
of group (iii). This shows the strong dependence of Be on metallicity, mainly for stars with $[\mathrm{Fe} / \mathrm{H}]<-1.6$ (Rebolo et al. 1992). The Li abundances in groups (ii) and (iii) reflect the presence of the Li plateau (Spite and Spite 1982) for metalpoor stars. The increasing Be abundance with metallicity and the constancy of the $\mathrm{Li}$ abundances can be interpreted in terms of pure galactic evolution and primordial nucleosynthesis, with no significant influence of mixing processes in the stellar interiors.
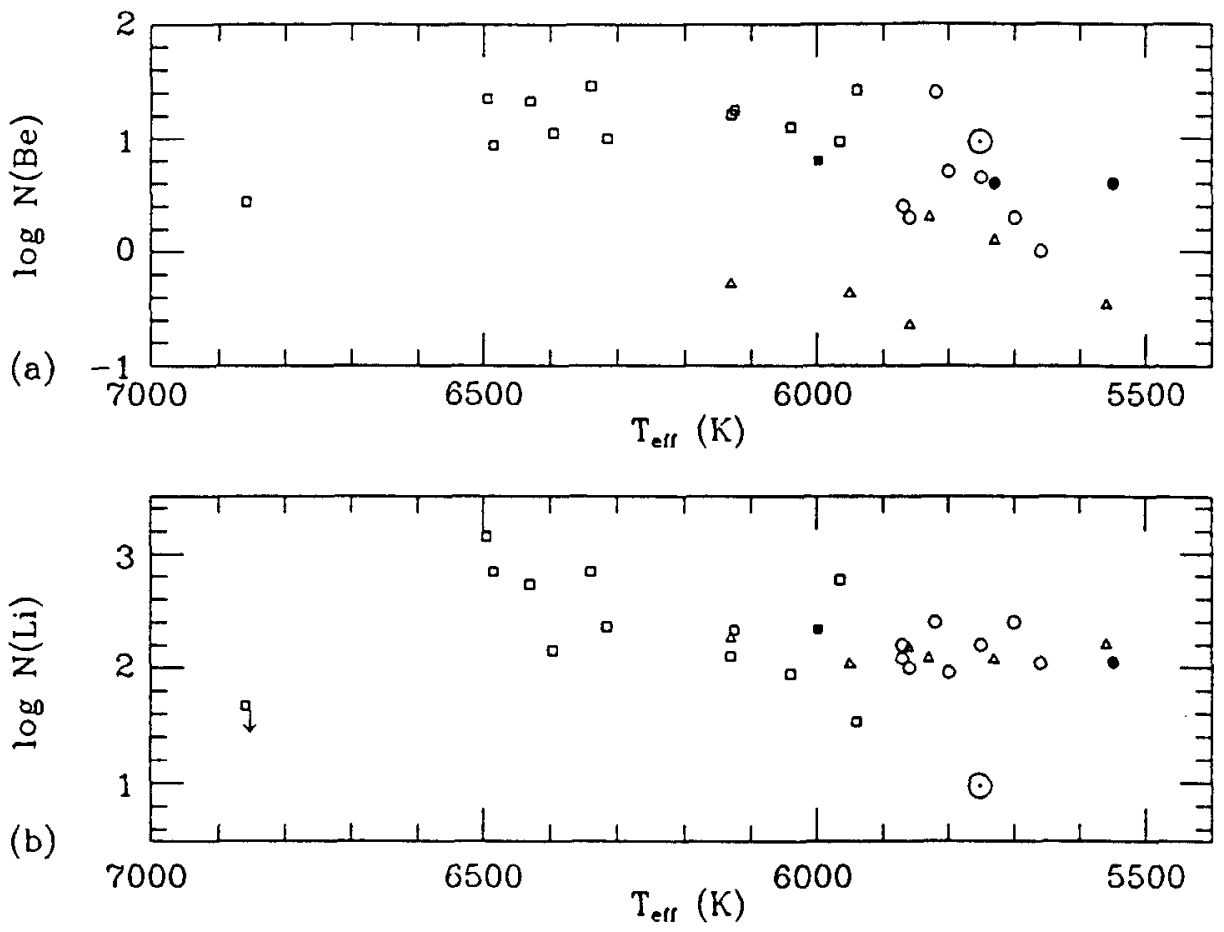

FIGURE I (a) Be abundances against effective temperature for unevolved stars divided in three metallicity bins: squares $[\mathrm{Fe} / \mathrm{H}]>-0.5$, circles $-0.5>[\mathrm{Fe} / \mathrm{H}]>-1.6$, triangles $[\mathrm{Fe} / \mathrm{H}]<-1.6$. Our stars are filled symbols. (b) $\mathrm{Li}$ abundances against effective temperature for the same stars.

\section{REFERENCES}

García López, R. J., and Spruit, H. C. 1991, Ap. J., ㅍ7, 268.

Rebolo, R., García López, R. J., Martín, E. L., Beckman, J. E., McKeith, C. D., Webb, J. K., and Pavlenko, Y. V. 1992, "On the Origin of the Elements", Eds. N. Prantzos, M. Cassé, and E. Vangioni-Flam, in press.

Spite, F., and Spite, M. 1982, Astr. Ap., 115, 357. 